\title{
Biochemical parameters of rat blood in the models of chronic heart failure and chronic kidney disease at the administration of nitric oxide donor
}

\author{
Ksenia Akhmadeeva*, Alisa Belova and Rufiia Karimova \\ Kazan State Academy of Veterinary Medicine, Physiology and Pathological Physiology Department, Kazan, 420029, Russia
}

\begin{abstract}
In modern veterinary medicine, the simultaneous occurrence of chronic heart failure and chronic kidney disease is often found. However, the cause and effect often exchange places, which creates great difficulties in the animals' treatment. Chlofusan acts on both systems. It improves cardiac and renal functions by means of providing cardioprotective and nephroprotective effects. Models of chronic heart failure and chronic kidney disease in rats provide important information on the pathophysiology of these diseases in other animal species, and the assessment of changes in the biochemical analysis of blood makes it possible to assess the state of the heart and kidneys in the study. In the course of research, the results of rat biochemical analysis were studied on models of chronic heart and kidney failure with the introduction of an exogenous nitric oxide donor. Chlofuzan contributes to a partial balance restoration of biochemical blood parameters in rats, which indicates the restoration of the mutual work of the heart and kidneys.
\end{abstract}

\section{Introduction}

A relationship has been established between the course of chronic heart failure $(\mathrm{CHF})$ and chronic renal disease (CKD), which is accompanied by a worsening prognosis of patient survival. [1-3]

$\mathrm{CHF}$ is a syndrome in which the heart does not provide sufficient blood circulation to meet the metabolic needs of the body, it accompanies almost all heart diseases. $[4,5]$

$\mathrm{CKD}$ is a syndrome resulting from an irreversible progressive decrease in renal function due to a decrease in the mass of functioning parenchyma concomitant with metabolic disorders and further development of pathology of a number of organs and systems. [6-9]

Modern medicine emphasizes the bi-directional nature of cardio-renal interaction and extensive interconnected disorders; the heart and kidneys, acting in tandem, regulate blood pressure, vascular tone, diuresis, natriuresis, intravascular volume homeostasis, peripheral tissue perfusion and oxygenation. [2, 3]

Very often veterinarians come across animals that have concomitant $\mathrm{CHF}$ and CKD. Cardiovascular disease is one of the causes of death when dogs or cats have terminal CKD. $[1,5]$ Both a decrease in glomerular filtration rate and a proteinuria is independent risk factors for the development of cardiovascular diseases. In some patients with severe renal artery stenosis, acute heart failure is clinically manifested due to volume and pressure overload. [10]

On the other hand, in the veterinary cardiologist's practice, cases of CKD are often found and resolved in the elimination of congestion in patients with ascites or pulmonary edema in heart failure. In addition, the decompensated stage of heart failure, cardiac ischemia and arrhythmia can lead to acute renal impairment due to insufficient filling of the renal artery and a decrease in renal blood flow, secondary to low cardiac output. A change in the processes that regulate cardiac and renal function is called cardiorenal syndrome. $[1,5,6,11]$

Cardiorenal syndrome is a pathophysiological disorder of the heart and kidneys in which one organ's acute or chronic dysfunction leads to the other's acute or chronic dysfunction. Thus, cardiorenal syndrome includes acute and chronic disorders in which the heart and kidneys can be the primary affected organ. [3, 12]

Normal cardiorenal relationships include the kidneys, which control the volume of extracellular fluid by regulating sodium excretion and reabsorption, and the heart, which controls systemic hemodynamics. This model's central links are rennin-angiotensin-aldosterone system (RAAS), endothelium-dependent factors and their antagonists. When one of the organs is damaged, RAAS and the sympathetic nervous system are activated, endothelial dysfunction and chronic systemic inflammation develop, a vicious circle is formed in which the combination of cardiac and renal dysfunction leads to an accelerated decrease in the each organ's functional ability and remodeling of the myocardium, vascular wall and renal tissue. [3, 11, 12]

The coexistence of CHF and CKD in the same animal has an extremely poor prognosis. Diagnosis of these two pathologies is very difficult for each syndrome separately, and even more complicated in their combination. Treatment of CKD and CHF is often

Corresponding author: theavess@gmail.com 
symptomatic and ineffective, which leads to the animal's early death from these pathologies.

The use of nitric oxide donors is due to the fact that nitric oxide (NO) in the heart supports vasodilation, regulates blood flow and controls basal blood pressure [14-17]. In kidneys it controls renal and glomerular hemodynamics, expands afferent and efferent arterioles, increases glomerular filtration rate, and inhibits transport sodium ions and increases its excretion, is involved in the ion exchange's regulation. $[6,7,18]$ Chlofusan is an exogenous donor of nitric oxide, which has a pronounced effect on the functional state of the nitroxidergic body's system. Clofusan a chlorosubstituted compound of the benzofuroxan series that activates the nitroxidergic system. [19]

The purpose of the work was to study the biochemical parameters of rat blood in models of chronic heart failure and chronic kidney disease with the introduction of a nitric oxide (II) donor. Research objectives:

1. to study the effect of Chlofusan on the biochemical parameters of rat blood in the CHF model

2. to study the effect of Chlofusan on the biochemical parameters of rat blood in the CKD model

3 . to compare the degree of influence of Chlofusan on CHF and CKD.

\section{Materials and methods}

The experiments were conducted at the Bauman Kazan State Academy of Veterinary Medicine in the Physiology and Pathological Physiology Department's laboratory from 2017 to 2019. In this experimental study, 25 male Wistar rats (230-250 g) were selected and divided into 5 groups randomly with 5 in each, as it will be described in more details below. Animals were housed on a 12 hours light-dark cycle and had free access to food and water. The groups were allocated as follows:

1 st group - intact,

2nd group - CHF-control,

3rd group - CHF-Chlofusan,

4th group - CKD-control,

5 th group - CKD-Chlofusan.

$\mathrm{CHF}$ was induced experimentally by administering the drug mesatone $(0.5 \mathrm{ml} / \mathrm{kg})$ for 21 days with subsequent physical exertion. [20] CKD was experimentally induced by a single injection of a 50\% aqueous solution of glycerol $(10 \mathrm{ml} / \mathrm{kg})$ into the hind limb's muscles. [21] Subsequently, rats with a models of CHF and CKD were given intragastric Chlofusan $(2 \mathrm{mg} / \mathrm{kg})$.

The material for the study was blood taken in a volume of $1 \mathrm{ml}$ from each rat from the tail vein. Biocemical analysis was performed using an automatic biocemical analyzer (Bi-An, Russia) with the biochemical kits (Olvex, Russia). The parameters included:

for CHF - Urea, Crea, AST, ALT, AST/ALT ratio, $\mathrm{LDH}, \mathrm{Na}, \mathrm{K}, \mathrm{Cl}$;

for CKD - Urea, Crea, AST, ALT, TP, Na, K, Cl.

The data were processed by statistical method using Student's t-test.

\section{Results and Discussion}

The biochemical parameters of rats in the CHF model are presented in table 1.

Table 1. Biochemical parameters of rat blood in the CHF model.

\begin{tabular}{|c|c|c|c|}
\hline Parameter & Intact & CHF-control & CHF-Chlofusan \\
\hline $\begin{array}{c}\text { Urea, mmol / } \\
\text { L }\end{array}$ & $5.71 \pm 0.23$ & $34.93 \pm 1.89^{*}$ & $21.19 \pm 1.66^{* *}$ \\
\hline $\begin{array}{c}\text { Crea, mmol / } \\
\text { L }\end{array}$ & $65.60 \pm 0.91$ & $141,3 \pm 4,99^{*}$ & $97,13 \pm 0,44^{* *}$ \\
\hline AST, U/L & $132 \pm 4.19$ & $335,98 \pm 8,27^{*}$ & $263,34 \pm 7,63^{* *}$ \\
\hline ALT, U/L & $127 \pm 3.79$ & $196,84 \pm 3,37^{*}$ & $154,28 \pm 1,83^{* *}$ \\
\hline $\begin{array}{c}\text { AST/ALT } \\
\text { ratio }\end{array}$ & $0.81 \pm 0.562$ & $1,70 \pm 0,09^{*}$ & $1,71 \pm 0,13$ \\
\hline LDH, U/L & $204 \pm 3.77$ & $228 \pm 14,086$ & $216 \pm 12,176$ \\
\hline Na, mmol / L & $103.80 \pm 9.84$ & $155.17 \pm 3,44 *$ & $120.82 \pm 2,97$ \\
\hline $\mathrm{K}, \mathrm{mmol} / \mathrm{L}$ & $4.34 \pm 0.24$ & $6,71 \pm 0,24 *$ & $4.98 \pm 0.18$ \\
\hline $\mathrm{Cl}, \mathrm{mmol} / \mathrm{L}$ & $87.81 \pm 1.29$ & $141.34 \pm 2.61 *$ & $129.11 \pm 5.73$ \\
\hline
\end{tabular}

* - significant as compared to the intact group $\mathbf{0}<0.05$

** - significant as compared to the CHF-control $\mathbf{0}<0.05$

In CHF-control AST increased by 2.55 times $(\mathrm{p} \leq 0.05)$, while ALT increased by only 1.54 times $(\mathrm{p} \leq 0.05)$ in comparison with the intact group. Given the change in the AST/ALT ratio by 2.09 times $(p \leq 0.05)$ in comparison with the intact group, this is a sign of cardiac pathology.

The LDH level in CHF-control was increased slightly by 1.11 times in comparison with the intact group, which means left-sided heart failure. An increase in Crea by 6.34 times $(\mathrm{p} \leq 0.05)$ and Urea by 2.15 times $(\mathrm{p} \leq 0.05)$ in CHF-control is a sign of developing renal failure. Elevated levels of $\mathrm{Na}$ by 1.5 times $(\mathrm{p} \leq 0.05), \mathrm{K}$ by 1.54 times $(p \leq 0.05)$ and $\mathrm{Cl}$ by 1.61 times $(p \leq 0.05)$ in CHFcontrol occurs due to RAAS's activation. The resulting angiotensin II has a pronounced vasoconstrictor effect, stimulates the release of norepinephrine and the aldosterone's secretion in the adrenal cortex, which leads to a salt's retention in the body.

The administration of Chlofusan reduces the concentration of AST and ALT by 1.27 times, keeping their ratio unchanged in comparison with CHF-control. A decrease in these indicators indicates an improvement in cardiac function, which can be used to relieve symptoms of heart failure in veterinary practice. A decrease in the concentration of Crea by 1.65 times and Urea by 1.45 times indicates an improvement in renal function, and a decrease in Na by 1.26 times, K by 1.57 times and $\mathrm{Cl}$ by 1.1 times means a decrease in RAAS's activity.

The administration of Chlofusan does not have a significant effect on LDH, this parameter changed 1.06 times.

The biochemical parameters of rats in the CKD model are presented in table 2 .

In CKD-control Crea and Urea concentrations were significantly increased by 14.2 times $(\mathrm{p} \leq 0.05)$ and by 13 times $(\mathrm{p} \leq 0.05)$ respectively, which indicates impaired 
renal function. These indicators are one of the most important parameters for meaning chronic renal failure.

The concentration of AST and ALT in the CKDcontrol significantly increased 2.15 times $(\mathrm{p} \leq 0.05)$ and 1.2 times $(\mathrm{p} \leq 0.05)$, respectively, proving the development of a pathological interdependent state of the heart and kidneys. An important factor in the differentiation of cardiac pathology is the indicator of the AST/ALT ratio, which also changes its values in CKDcontrol similar to CHF control. So, in CKD-control this parameter is 2.2 times $(\mathrm{p} \leq 0.05)$ higher than in the intact group, which means the concomitant development of heart failure and cardiorenal syndrome.

Table 2. Biochemical parameters of rat blood in the CKD model

\begin{tabular}{|c|c|c|c|}
\hline Parameter & Intact & CKD-control & $\begin{array}{c}\text { CKD- } \\
\text { Chlofusan }\end{array}$ \\
\hline Urea, mmol / L & $5.71 \pm 0.23$ & $74.91 \pm 1.43^{*}$ & $70.53 \pm 6.35^{* *}$ \\
\hline Crea, mmol / L & $65.60 \pm 0.91$ & $929.60 \pm 30.55^{*}$ & $661.20 \pm 68.09^{* *}$ \\
\hline AST, U/L & $132 \pm 4.19$ & $284.24 \pm 4.18^{*}$ & $249.33 \pm 3.72^{* *}$ \\
\hline ALT, U/L & $127 \pm 3.79$ & $156.84 \pm 2.27 *$ & $135.26 \pm 2.89^{* *}$ \\
\hline AST/ALT ratio & $0.81 \pm 0.562$ & $1.81 \pm 1.84^{*}$ & $1.83 \pm 1.1$ \\
\hline TP, g/L & $64.80 \pm 2.58$ & $66.40 \pm 0.91$ & $72.20 \pm 1.92$ \\
\hline Na. mmol/L & $103.80 \pm 9.84$ & $107.00 \pm 0.79$ & $121.40 \pm 1.53^{* *}$ \\
\hline $\mathrm{K}, \mathrm{mmol} / \mathrm{L}$ & $4.34 \pm 0.24$ & $4.84 \pm 0.47$ & $8.70 \pm 1.09^{* *}$ \\
\hline $\mathrm{Cl}, \mathrm{mmol} / \mathrm{L}$ & $87.81 \pm 1.29$ & $83.60 \pm 1.96$ & $85.40 \pm 1.35$ \\
\hline
\end{tabular}

* - significant as compared to the intact group $\mathbf{0}<0.05$

** - significant as compared to the CKD-control $\mathbf{0}<0.05$

In CKD-control Crea and Urea concentrations were significantly increased by 14.2 times $(\mathrm{p} \leq 0.05)$ and by 13 times $(\mathrm{p} \leq 0.05)$ respectively, which indicates impaired renal function. These indicators are one of the most important parameters for meaning chronic renal failure.

The concentration of AST and ALT in CKD-control significantly increased 2.15 times $(\mathrm{p} \leq 0.05)$ and 1.2 times $(\mathrm{p} \leq 0.05)$, respectively, proving the development of a pathological interdependent state of the heart and kidneys. An important factor in the differentiation of cardiac pathology is the indicator of the AST/ALT ratio, which also changes its values in CKD-control similar to CHF control. So, in CKD-control this parameter is 2.2 times $(\mathrm{p} \leq 0.05)$ higher than in the intact group, which means the concomitant development of heart failure and cardiorenal syndrome.

In contrast to the $\mathrm{CHF}$ model, in $\mathrm{CKD}$-control group the changes in $\mathrm{Na}$ were 1.03 times, K 1.11 times, and $\mathrm{Cl}$ 0.94 times $(\mathrm{p} \leq 0.05)$ insignificant. Changes in TP are also not significant, which indicates the absence of a significant inflammatory process.

With the introduction of an exogenous donor of nitric oxide Chlofusan, the concentrations of Crea is changed by 1.1 times $(p \leq 0.05)$ and Urea by 1.4 times $(p \leq 0.05)$ compared with the CKD-control. Compared with similar changes in CHF-Chlofusan, the restoration of renal function in rats in the CKD model is less pronounced than in the $\mathrm{CHF}$ model and concomitant renal failure (Fig. 1).

In CKD-Chlofusan, the decrease in AST was 1.14 times $(p \leq 0.05)$ and 1.16 times $(p \leq 0.05)$ less pronounced than in CHF-Chlofusan (Fig. 2), however, changes in these parameters were less pronounced than in $\mathrm{CHF}$ control. At the same time, the administration of Chlofusan does not change the AST/ALT ratio in both models.

After Chlofusan's administration the concentration of $\mathrm{Na}$ increases in 1.1 times $(\mathrm{p} \leq 0.05)$ and $\mathrm{K}$ in 1.8 times $(\mathrm{p} \leq 0.05)$, which means an increase of RAAS's activity as a result of Chlofusan acts on the rat organism in the CKD model (fig. 3).

Thus, impaired renal function as a result of chronic renal failure leads to the adverse effects to the rats which have heart failure and vice versa. Such interconnected disorders are caused by a commonality of the mechanisms underlying the hemodynamic kidneys' and heart's failures. The administration of donor a nitric oxide - Chlofusan leads to partial relief of the course of chronic renal failure and heart failure, restores the biochemical parameters of blood. For animals with $\mathrm{CHF}$ and CKD, it is necessary to diagnose cardiorenal syndrome as early as possible. The administration of nitric oxide (II) donors can be a good tool to prevent the diseases' progression, as well as to prevent the development of kidney damage in sick animals with heart failure.

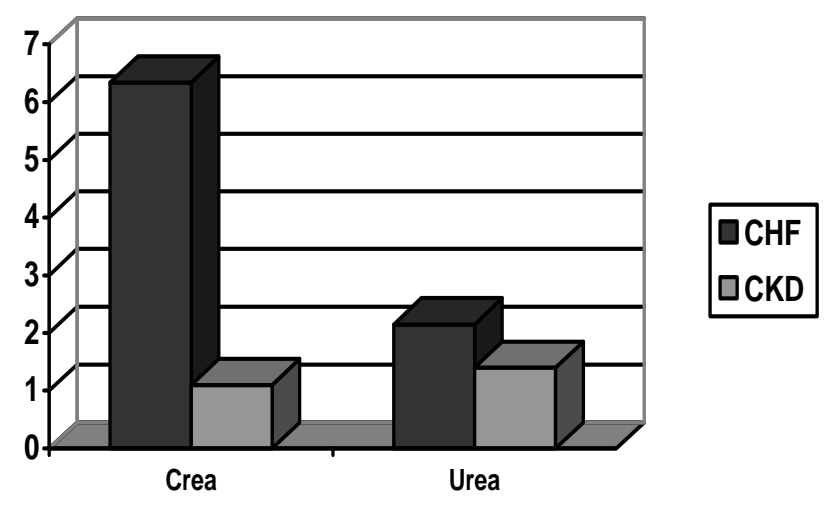

Fig. 1. Changes in renal parameters in CHF and CKD models after administration of chlorofusan. 


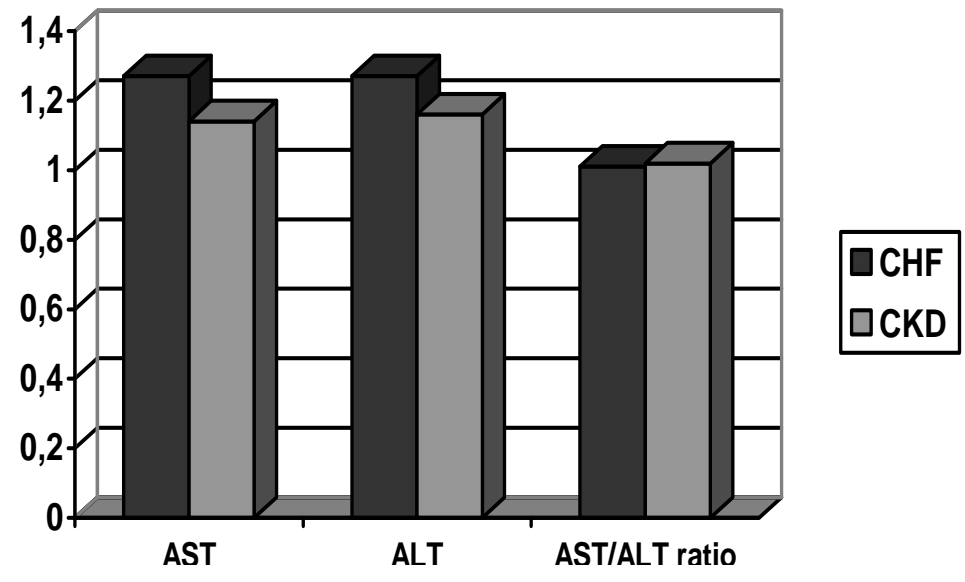

Fig. 2. Changes in cardiac parameters in CHF and CKD models after administration of Chlofusan.

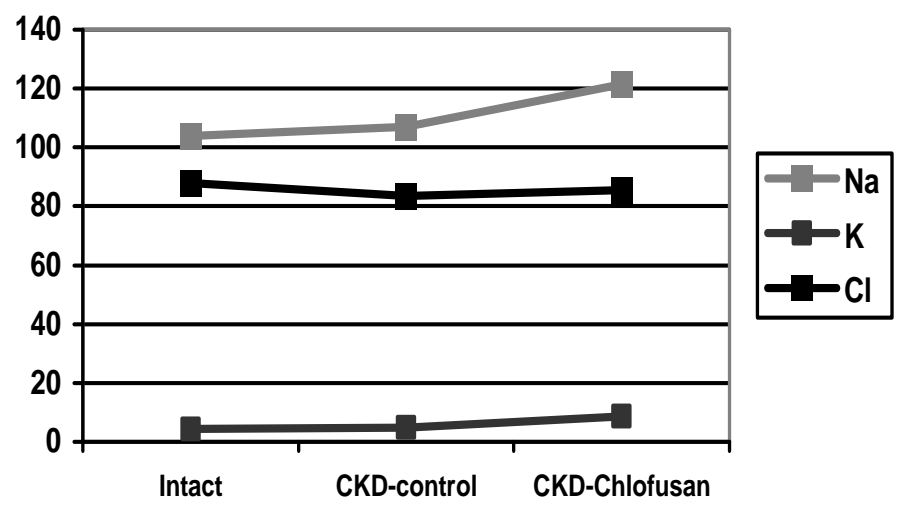

Fig. 3. Changes salt's parameters in CKD models

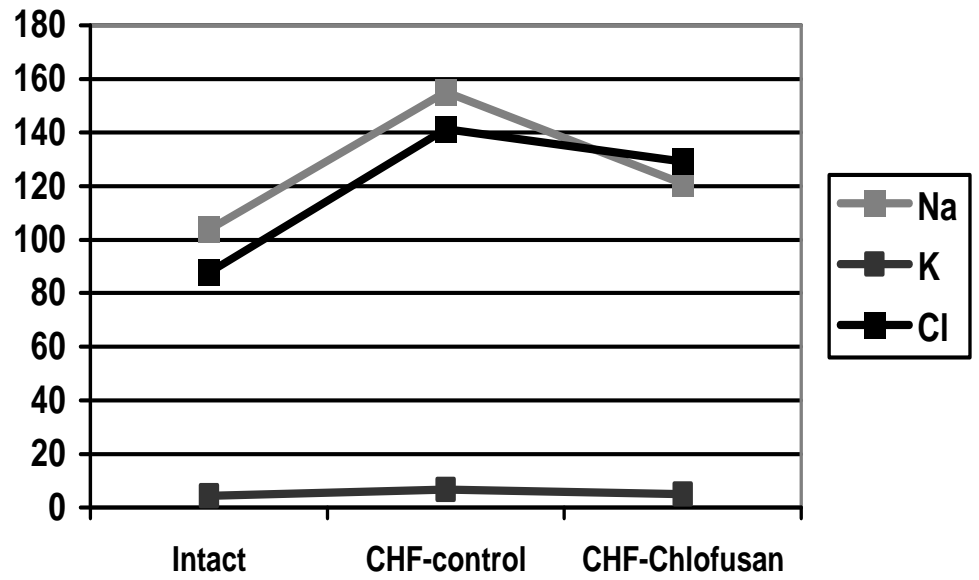

Fig. 4. Changes salt's parameters in CHF models

\section{Conclusion}

CHF causes a significant increase in AST and a slight ALT, the AST/ALT ratio in CHF is always greatly increased, which allows us to differentiate cardiac pathology from hepatic pathology. Changes in LDH indicate left-side heart failure, which is consistent with an increase in Crea and Urea. The accumulation of salts in the blood is accompanied by activation of the RAAS.
CKD causes a significant increase in Crea and Urea, an increase in these parameters is the main sign of kidney dysfunction. CKD has an effect on cardiac function, increasing AST and slightly ALT, as well as the AST/ALT ratio.

Chlofusan has a positive effect on the balance of biochemical parameters of the rats' blood. The established facts require additional chronic experiments, since at the moment it is not known whether the effect of the action of nitric oxide donors will persist for a long time or is it a temporary phenomenon, resulting from body compensatory mechanisms. 


\section{References}

1. J.L. Pouchelon, C.E. Atkins, C. Bussadori et al., J. Small Anim. Pract. 56, 9 (2015)

2. S. Hadjiphilippou, S.P. Kon, J. Soc. Med. 109, 1 (2016)

3. I.S. Anand, Clin. J. Am. Soc. Nephrol. 8, 10 (2013)

4. A.I. Girfanov, K.E. Akhmadeeva, R.G. Karimova, Sci. Notes KSAVM 239(III), 16 (2019)

5. A. Agrawal, M. Naranjo, N. Kanjanahattakij, J. Rangaswami, S. Gupta, Heart Fail Rev. 24, 4 (2019)

6. J. Passauer, F. Pistrosch, E. Büssemaker, Kidney Int. 67, 5 (2005)

7. J. Kaur, B.E. Young, P.J. Fadel, Int. J. Mol. Sci. 18, 8 (2017)

8. J. Zhong, H.C. Yang, F.B. Fogo, Am. J. Physiol. Renal. Physiol. 312, 3 (2017)

9. A. Shirai, O. Yamazaki, S. Horita, M. Nacamura et al., J. of the Amer. Society of Nephrol. 25, 7 (2014)

10. B.J. Freda, Cleve Clin. J. Med. 85, 5 (2018)

11. M. Liu, X.C. Li, L. Lu, Y. Cao et al., Eur. Rev. Med. Pharmacol. Sci. 18, 19 (2014)
12. H. Takahama, M. Kitakaze, Am. J. Physiol. Heart Circ. Physiol. 313, 4 (2017)

13. R. Bonafede, W. Manucha, Clin. Investig. Arterioscler. 30, 2 (2018)

14. M.T. Ziolo, M.J. Kohr, H. Wang, J. Mol. Cell. Cardiol. 45, 5 (2008)

15. H. Strijdom, N. Chamane, A. Lochner, Cardiovasc. J. Afr. 20, 5 (2009)

16. M. Totzeck, U.B. Hendgen-Cotta, T. Rassaf, NitriteNitric Oxide Signaling and Cardioprotection Adv. Exp. Med. Biol. 982, 335-346 (2017)

17. I. Cuadrado, B. Castejon, A.M. Martin, M. Saura, P. Reventun-Torralba, J.L. Zamorano, C. Zaragoza, PLoS One 11, 9 (2016)

18. J.B. Rosenbaek, S.A. Therwani, J.M. Jensen, F.H. Mose, C. Wandall-Frostholm, E.B. Pedersen, J.N. Bech, Am. J. Physiol. Renal. Physiol. 313, 2 (2017)

19. R.G. Karimova, T.V. Garipov, VMI-REAVIZ 2, 6-11 (2011)

20. Yu.V. Liskova, S.P. Salikova, A.A. Stadnikov, Morpholog. Newsletter 1, 46-53 (2014)

21. I.V. Borisova, S.Yu. Shtrigol, Medline. RU. 5, 36 (2004) 